\title{
红霉素的生物合成与组合生物合成
}

\author{
吴杰群 ${ }^{a, b}$ 刘 文 ${ }^{b}$ 张嗣良 ${ }^{*, a}$ \\ $\left({ }^{a}\right.$ 华东理工大学 生物反应器工程国家重点实验室 上海 200237) \\ ( ${ }^{b}$ 中国科学院上海有机化学研究所 生命有机化学国家重点实验室 上海 200032)
}

\begin{abstract}
摘要 组合生物合成在篎选和发展新型药物方面日益被生物、化学和医药界所关注. 红霉素作为组合生物合成发展的 模式化合物一直是人们研究的热点. 概述了红霉素的生物合成机制及近年来在此基础上采用组合生物合成获得红霉素 衍生物的研究进展，并对此方面存在的问题、应用前景作了展望.
\end{abstract}

关键词 红霉素; 生物合成; 组合生物合成

\section{Biosynthesis and Combinatorial Biosynthesis of Erythromycin}

\author{
Wu, Jiequn ${ }^{a, b} \quad$ Liu, Wen ${ }^{b} \quad$ Zhang, Siliang ${ }^{*, a}$ \\ ( ${ }^{a}$ State Key Laboratory of Bioreactor Engineering, East China University of Science and Technology, Shanghai 200237) \\ ( ${ }^{b}$ State Key Laboratory of Bio-Organic and Natural Products Chemistry, Shanghai Institute of Organic Chemistry, \\ Chinese Academy of Sciences, Shanghai 200032)
}

\begin{abstract}
Combinatorial biosynthesis plays a growing role of drug discovery and development in the fields of biology, chemistry and medical sciences. Erythromycin, as the model molecule, has long been appreciated for the investigations into the biosynthesis of natural products and their associated structural diversity by pathway engineering. In this paper the progress regarding erythromycin in biosynthesis and combinatorial biosynthesis is reviewed. The problems and application prospects are also discussed.
\end{abstract}

Keywords erythromycin; biosynthesis; combinatorial biosynthesis

在化学结构方面具有丰富多样性的天然产物，一直 是人类治疗疾病的主要药物. 寻找和开发新的天然产物 药物, 也是化学界与医药界关注的重点领域. 20 世纪 20 年代末弗莱明偶然从青霉菌里发现青霉素, 不仅标志着 人类历史上第一个抗生素的诞生，同时也掀起了人们从 微生物代谢产物中寻找药物的大潮. 在这期间, 不仅成 千上万的抗生素被发现, 具有其它生物活性的微生物天 然产物如抗真菌的两性霉素(Amphotericin B)、抗肿瘤的 柔红霉素(Daunorubicin)及免疫抑制剂 FK506 等也被发 展成为各式各样的药物. 到 20 世纪末已发现的天然产 物中有 $2 / 3$ 来源于微生物 ${ }^{[1]}$. 然而随着这些天然产物特 别是抗生素的广泛使用, 细菌对它们的耐药性也越来越 强. 万古霉素曾经很长一段时间被认为是抗耐甲氧西林 金黄色葡萄球菌的最后一道防线. 最近耐万古霉素的 “超级细菌”也出现, 显然细菌对抗生素产生耐药性是不 可逆转的 ${ }^{[2]}$. 人类对感染性疾病面临无药可治的困境, 寻找和开发新的抗生素显然成为人类迫不急待的任务.
然而，新型抗生素发现的 “黄金时代” 已经过去. 近几 十年来从常规环境中篮选的活性天然产物大多和以前 发现的抗生素具有相同或类似的结构, 或者具有相同的 抗菌机制 ${ }^{[3]}$. 从极端环境微生物代谢产物中篮选新的天 然产物, 成为当前研究的一个方向 ${ }^{[4]}$. 人们开发新来源 活性天然产物的同时, 往往忽视了一个已经存在的活性 天然产物宝库. 据估计目前大概有 $10^{5}$ 种的抗生素被发 现，但是只有 $10^{2}$ 种的抗生素被用于临床 ${ }^{[2]}$, 其余的抗生 素因为毒副作用、或者水溶性差、或者抑菌活性不高等 原因而不能成为临床药物. 以活性天然产物为母体进行 化学结构修饰合成新的衍生物, 与从环境中随机篮选新 的天然产物相比更具有针对性，因此也成为目前新药发 展的重要途径之一 ${ }^{[5]}$. 由于大部分天然产物的结构十分 复杂, 采用化学方法进行合成或结构修饰往往非常困 难 ${ }^{[6]}$. 近年来发展的组合生物合成(Combinatorial biosynthesis)技术为复杂天然产物及其类似物的获得提供 了一条生物合成的方法 ${ }^{[7]}$. 在掌握了某个天然产物的生

*E-mail: siliangz@ecust.edu.cn

Received October 8, 2011; revised January 16, 2012; published online February 8, 2012. 
物合成机制后, 我们可以特异性地从遗传水平上操纵天 然产物的代谢途径, 以此获得基因重组菌株来合成特定 的天然产物及其类似物; 或者从基因水平对天然产物的 生物合成途径进行重组, 建立结构复杂的天然产物类似 物库, 以利于从中发现和发展更具应用价值的药物. 目 前, 组合生物合成无论是在学术研究还是工业领域, 对 于新药开发和生产的巨大影响力正逐渐为科学家们所 认同. 它们的成功运用, 关键是在基因和蛋白功能水平 上认识和理解复杂天然产物的生物合成及其调控机制, 这是对代谢途径进行针对性改造的分子和生化基础.

红霉素是一类用于治疗革兰氏阳性细菌感染的广 谱大环内酯类抗生素, 最早于 1952 年从红色糖多狍菌 (Saccharopolyspora erythraea, 当时命名为红霉素链雱 菌 Streptomyces erythreus) 的发酵产物里分离得到 ${ }^{[8]}$. 红 霉素 A (1) 是发酵液的主要产物, 结构上由一个十四元 环内酯及在大环内酯上接合两个糖基组成. 目前以红雩 素 A 为基础开发出来的第二代红霉素如阿齐霉素(2)、 克拉霉素(3)、罗红霉素(4)以及第三代红霉素泰利霉素 (5)等是临床上广泛使用的抗生素之一 ${ }^{[9]}$. 这些对红霉素 进行结构修饰所产生的新一代药物不仅能提高它的临 床疗效, 甚至对原本红霉素的抗菌谱也有较大的扩 展 ${ }^{[10]}$. 显然, 通过组合生物合成对红霉素进行结构改造 在新药开发上具有巨大的潜力. 目前关于红霉素的生物 化学方面的研究有许多报道, 包括生物合成途径的阐 明 $^{[11]}$ 、关键酶结构的鉴定和催化机制的推导 ${ }^{[12 \sim 14]}$, 以及 产生菌的全基因组测序等 ${ }^{[15]}$, 这为红霉素的组合生物 合成提供了丰富的理论基础. 也正因为如此, 红霉素成 为当今组合生物合成研究最为热点的模式化合物. 本文 综述了近年来红霉素生物合成机制的相关研究以及其 组合生物合成取得的进展.

\section{1 红霉素的生物合成机制}

在目前发现的具有良好生物活性的天然产物中, 聚 酮类化合物占据了很大的比例. 这类化合物是以小分子 羧酸为前体, 通过聚酮合酶(polyketide synthase, PKS)催 化合成的. 根据催化模块是否重复利用以及功能域的不 同, PKS 可分为 I 型、 II 型和 III 型 ${ }^{[16]}$. 红霉素属于 I 型 PKS 合成的聚酮化合物. I 型 PKS 聚酮合酶是一个巨大 的多功能蛋白. 该蛋白包含有多个模块(Module), 每个 模块由不同功能的结构域组成. 这些结构域按功能可分 为: 酰基转移酶(acyltransferase, $\mathrm{AT}$ ), 酰基载体蛋白 (acyl carrier protein $\mathrm{ACP}$ ), $\beta$-酮基硫酯合成酶 (ketosynthase, KS), $\beta$-酮基还原酶(ketoreductase, KR), 脱 水酶 (dehydratase, DH) 和烯醇还原酶 (enoylreductase, ER)等功能域.
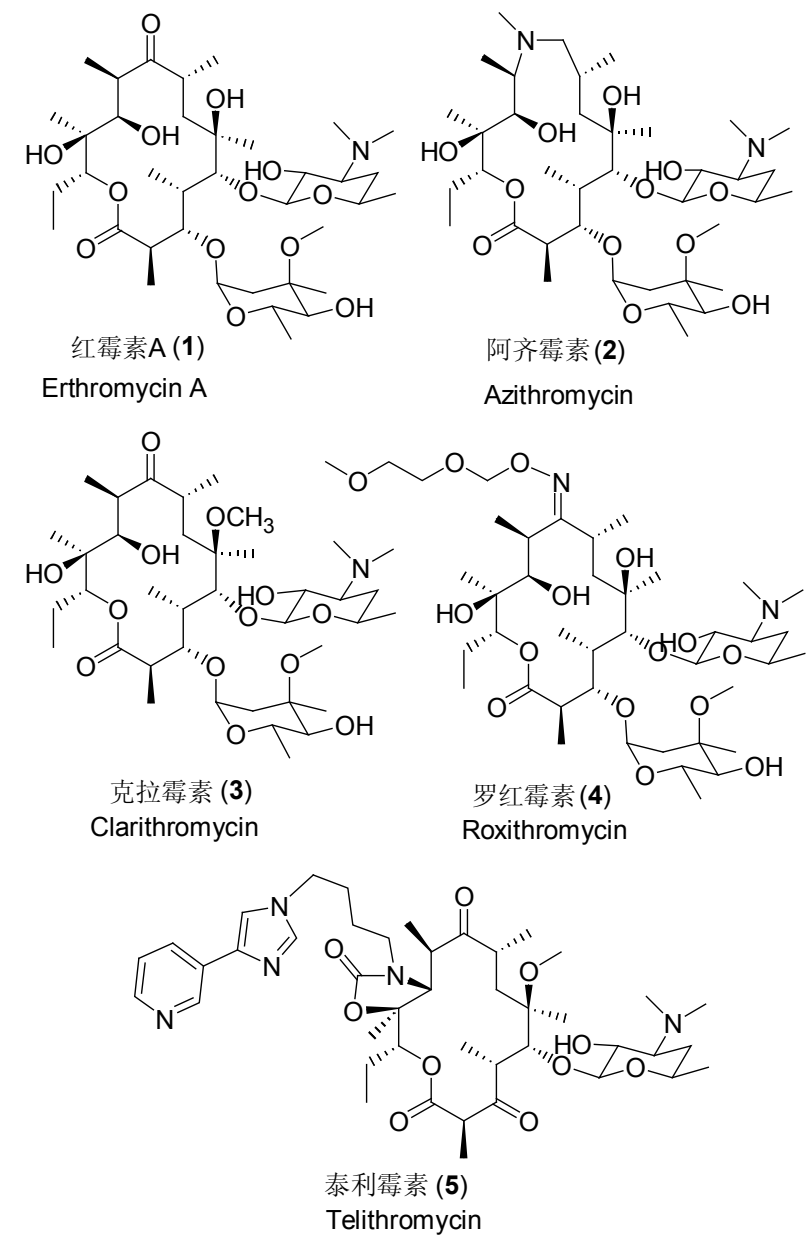

红霉素的生物合成的起始步骤是由 1 分子的丙酰辅 酶 $\mathrm{A}$ 和 6 分子的甲基丙二酸单酰辅酶 $\mathrm{A}$ 通过 I 型 PKS 聚酮合酶复合酶系缩合而成 6-脱氧红霉内酯(6-deoxyerythronolide B, 6-dEB, 6) ${ }^{[17]}$. 该复合酶系包含三个酶蛋 白亚基: DEBS1, DEBS2 和 DEBS3, 共由 1 个起始模块 和 6 个延伸模块组成 ${ }^{[18]}$. 6-脱氧红霉内酯的合成首先通 过起始模块中的酰基转移酶 $\mathrm{AT}_{0}$ 特异性识别丙酰辅酶 $\mathrm{A}$, 将其转移到酰基载体蛋白 $\mathrm{ACP}_{0}$ 上作为合成的起始 单元. 随后起始单元被转移到第一个延伸模块的 $\beta$-酮基 硫酯合成酶 $\mathrm{KS}_{1}$ 上, 同时第一个延伸模块中的 $\mathrm{AT}_{1}$ 特异 识别甲基丙二酸单酰辅酶 $\mathrm{A}$ 为底物并将其转移到第一 个延伸模块的酰基载体蛋白 $\mathrm{ACP}_{1}$ 上. 在 $\mathrm{ACP}_{1}$ 上 $\mathrm{KS}_{1}$ 催化起始单元丙酰基与延伸单元物甲基丙二酸单酰基 缩合形成 $\beta$-酮完成第一轮延伸. 其它 5 个延伸模块采用 类似的机理依次线性完成缩合. 在每一轮延伸后, 主链 骨架都增加两碳单元, 最终形成一个十四碳骨架的单元 链. 在 DEBS3 的羟基端有一个硫酯酶域(Thioesterase, $\mathrm{TE}$ ), 负责将合成的长链脂肪酸从 PKS 上水解下来环化 形成一个十四元环的化合物一 - 6 -脱氧红霉内酯(图 1). 从该合成过程可以看出 $\beta$-酮基硫酯合成酶 KS、酰基转 移酶 $\mathrm{AT}$ 和酰基载体蛋白 $\mathrm{ACP}$ 是每个延伸模块所必须 
的基本功能域，模块含有的 $\beta$-酮基还原酶 $\mathrm{KR}$ 、脱水酶 $\mathrm{DH}$ 和烯醇还原酶 ER 等功能域, 会对单元链进行修 饰 ${ }^{[18]}$.

在合成 6-脱氧红霉内酯后, 红霉素的生物合成进入 了大环内酯环的后修饰阶段. 内酯环的后修饰首先是通 过一个细胞色素 P450 系的羟化酶(EryF)在 6-脱氧红霉 内酯大环骨架 C-6 位接上一个羟基形成红霉内酯 (erythronolide B, EB，7). 然后再在红霉内酯大环骨架 C-3 羟基的位置上由糖基转移酶(EryBV)连接上一个 $L-$ 碳霉糖(mycarose)形成 3-O-碳霉糖基红霉内酯(3- $\alpha$-mycarosyl erythronolide B, MEB, 8). 紧接着又通过另外一 个糖基转移酶(EryCIII) 在 C-5 羊基的位置上连接一个 $D$ 德胺糖(desosamine), 从而形成红霉素合成中间代谢产 物中第一个有生物活性的物质红霉素 D. 红需素 D 在一 个 C-12 羟化酶(EryK)的催化下合成红霉素 C, 然后红霉 素 C 在一个由 SAM 依赖的甲基化酶(EyG)作用下在 C-3 的碳需糖上加上一个甲基合成最终产物红霉素 A. 此 外, 红霉素 $\mathrm{D}$ 也可以先在甲基化酶(EryG)的作用下在 C-3 碳霉糖的糖基上得到一个甲基合成红霉素 $\mathrm{B}$, 再通
过羟化酶(EryK)在 C-12 位置羟化合成红霉素 A. 此条途 径催化效率较低, 为红霉素 $\mathrm{A}$ 合成的副途径 ${ }^{[19]}$ (图 2). 在红霉素工业生产的发酵液里有大量中间产物红霉素 $\mathrm{B}$ 和红霉素 $\mathrm{C}$ 积累 ${ }^{[20]}$. 红霉素 $\mathrm{B}$ 和红霉素 $\mathrm{C}$ 的抗菌活性 要比红霉素 $\mathrm{A}$ 低, 毒副作用却比红霉素 $\mathrm{A}$ 大, 在红霉素 工业生产过程中属于很难除去但又必须除去的杂质. 本 实验室通过增加、优化红色糖多孢菌 eryK 和 eryG 基因 拷贝数来调控对应酶的活性, 成功地在细胞内把红霉素 $\mathrm{B}$ 和红霉素 $\mathrm{C}$ 完全转化为红霉素 $\mathrm{A}$, 为红霉素的工业生 产带来了便利 ${ }^{[21,22]}$.

\section{2 红霉素的组合生物合成研究}

红霉素的生物合成可以简单地归为内酯环的形成 和内酯环的后修饰两部分. 对红霉素的组合生物合成研 究，也可以分为两个方向：一个方向是对合成红霉素大 环内酯的 PKS 进行遗传操作，改变大环内酯的结构; 另 一个方向是对红霉素合成的后修饰途径进行改造，合成 具有不同糖基的红霉素类似物.

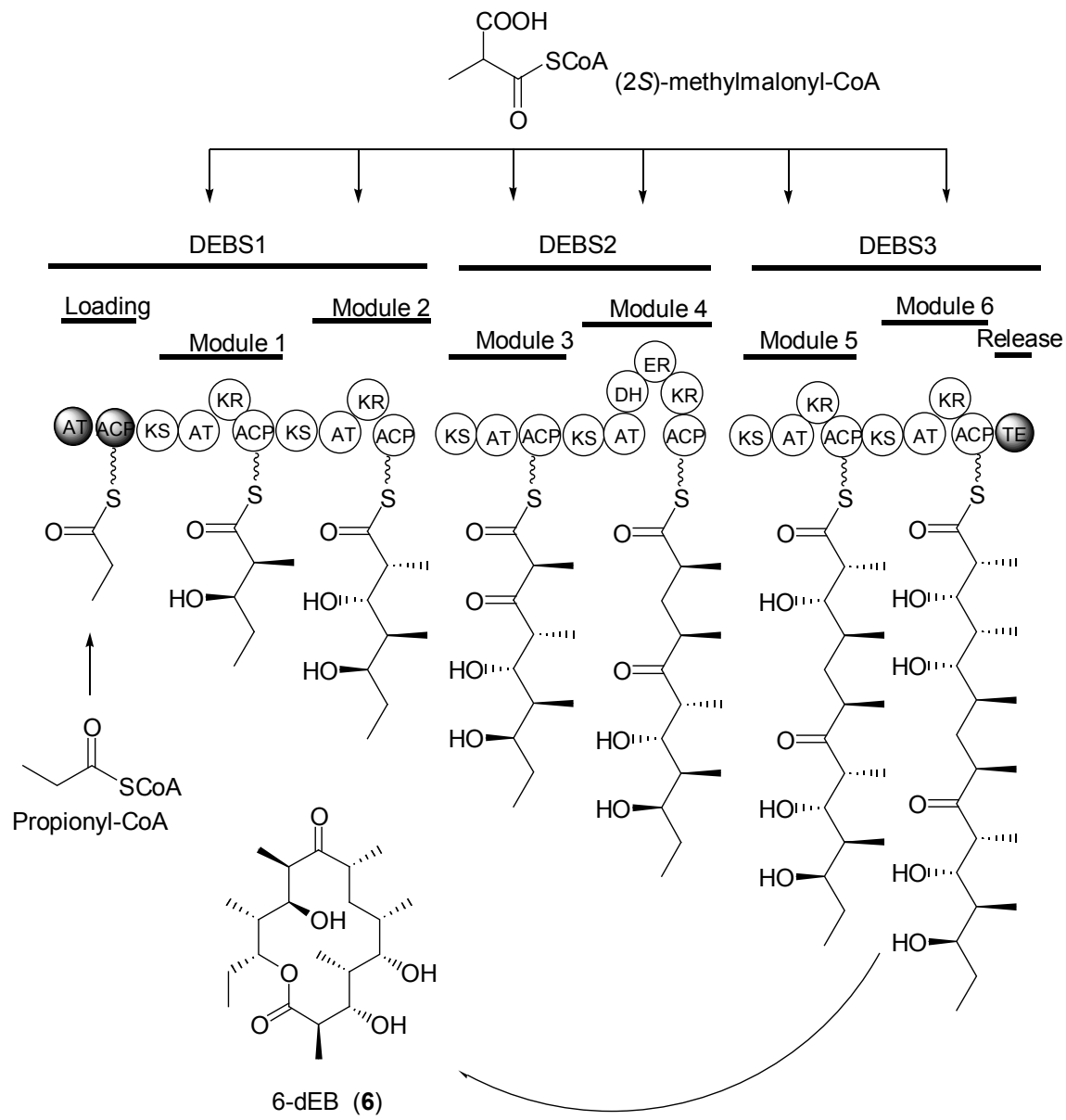

图 1 红霉素 DEBS 蛋白的模块组成

Figure 1 Modular organization of deoxyerythronolide B synthase (DEBS) 


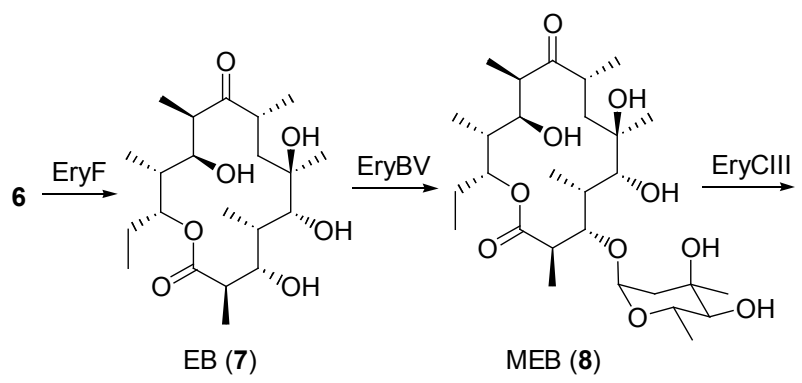

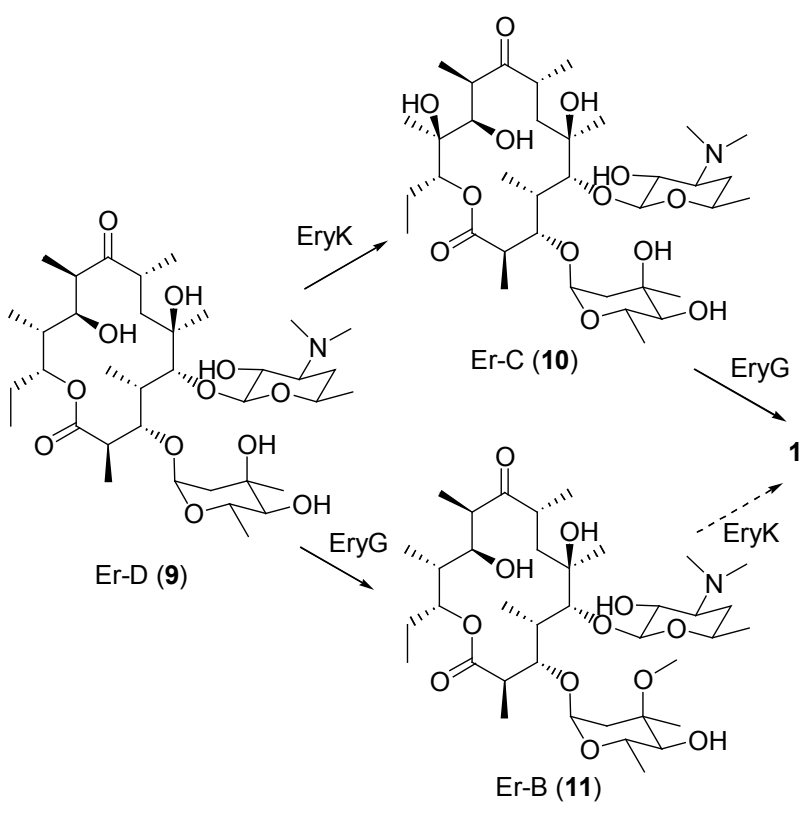

图 2 合成红霉素 $\mathrm{A}$ 的后修饰步骤

Figure 2 Post-PKS pathway in erythromycin A biosynthesis

I 型 PKS 聚酮合酶是以模块组合形式存在, 每一模 块含有一套非重复使用的催化功能域, 催化反应与功能 域呈一一对应的线性关系. 在 PKS 的亚基、模块或者结 构域中任何一点功能上的改变, 都将对大环内酯合成线 性顺序反应中的某一步产生影响, 使合成的大环内酯结 构发生细微的变化, 最终将产生结构类似但又不完全相 同的聚酩化合物. 目前对红霉素大环内酯组合生物合成 研究主要集中在模块功能域的改变上, 常用的分子生物 学操作手段包括结构域的点突变失活、替换, 模块的替 换或插入，不同蛋白亚基之间的杂交等 ${ }^{[23]}$.

\section{1 酰基转移酶 AT}

酰基转移酶 AT 负责特异性识别底物, 替换识别不 同底物的模块, 可以使不同的底物掺入内酯环的合成 中. 在 6-脱氧红霉内酯合成过程中 6 个延伸模块的酰基 转移酶 AT 都特异识别甲基丙二酸单酰辅酰 A 为底物, 将特异识别丙二酰辅酶 A 的 AT 结构域分别替换模块 1 到模块 6 的 AT 结构域, 将分别产生在内酯环 C-12, C-10, C-8, C-6, C-4 和 C-2 位置缺少甲基的红霉素结构 类似物 $12 \sim 17^{[24 \sim 27]}$.

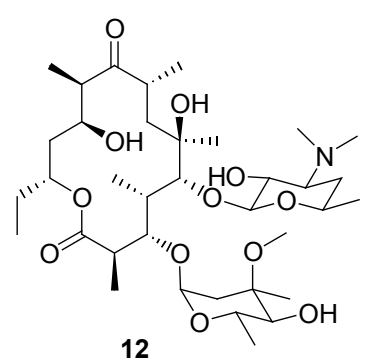

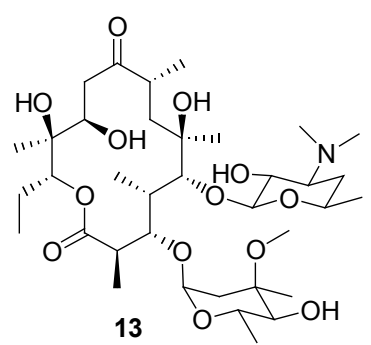

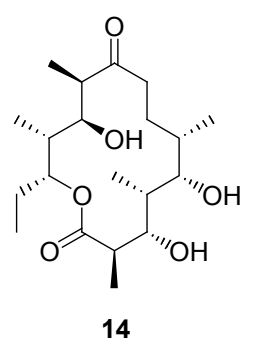

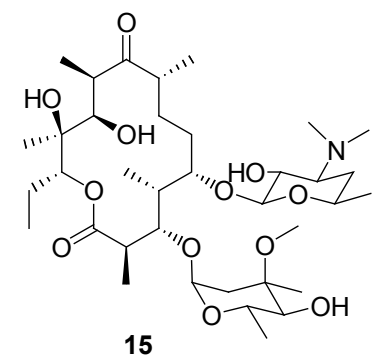<smiles>CC[C@H](OC(=O)C(C)[C@@H](O)C[C@@H](O)[C@@H](C)C[C@@H](C)O)[C@@H](C)C(=O)[C@H](C)C(=O)C(C)C</smiles><smiles>CC[C@H](OC(=O)C[C@@H](O)[C@@H](C)[C@H](O)[C@@H](C)C[C@@H](C)C(=O)[C@H](C)O)[C@@H](C)C(=O)[C@H](C)O</smiles>

17

同样采用一个识别乙基丙二酰辅酶 A 为底物的 AT 功能域(来自 Niddamycin 的 PKS 模块 7)替换红霉素 PKS 模块 5 , 则可获得 C-6 位上的乙基取代类似物 $18^{[28]}$.

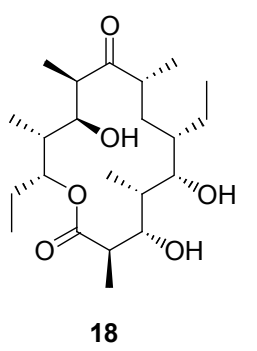

对于红霉素聚酮合酶 PKS 起始模块中的酰基转移 酶 AT, 其本身在红色糖多狍菌体内选择性识别丙酰辅 酶 A 为底物. 而阿维菌素合成 PKS 中起始模块的 AT 具 有广泛底物选择性的, 把后者的 AT-ACP 区域替换原本 红霉素合成 PKS 所对应的区域后，杂合的 PKS 能够识 别异丁酰辅酶 $\mathrm{A}$ 或者 2-甲基丁酰辅酶 $\mathrm{A}$ 为起始单元, 合 成 C-13 位上带异丙基或 2-丁基的红霉素结构类似物 19 24 ${ }^{[29]}$. 红霉素 PKS 的起始模块仅含有 AT-ACP 结构 域，但是许多 I 型 PKS 聚酮化合物如泰乐菌素 $(\text { tylosin })^{[30]}$ 、苦霉素(pikromycin) ${ }^{[31]}$ 、多杀菌素(spinosyn $)^{[32]}$ 等的起始模块 AT-ACP 结构域的前端还含有 KSQ 功能域. 这种结构单元的起始模块 AT 首先识别的是二 
酸单酰辅酶 $\mathrm{A}$, 结合到酰基载体蛋白 $\mathrm{ACP}$ 后并不是直接 转移到延伸模块的 $\mathrm{KS}$ 结构域中, 而是由其自身模块的 酮基硫酯合成酶 KSQ 结构域催化脱羧, 再转移到第一 个延伸模块的酮基硫酯合成酶 KS1 上进行下一步的合 成(图 3).

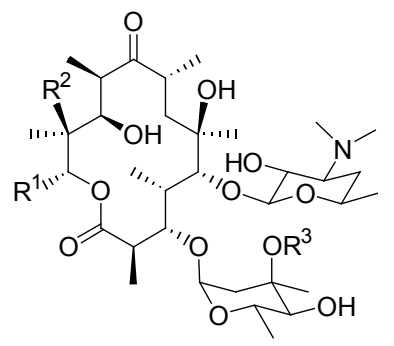

$19 \mathrm{R}^{1}=$ iso-Propyl, $\mathrm{R}^{2}=\mathrm{OH}, \mathrm{R}^{3}=\mathrm{CH}_{3}$

$20 \mathrm{R}^{1}=$ iso-Propyl, $\mathrm{R}^{2}=\mathrm{H}, \mathrm{R}^{3}=\mathrm{CH}_{3}$

$21 \mathrm{R}^{1}=$ iso-Propyl, $\mathrm{R}^{2}=\mathrm{H}, \mathrm{R}^{3}=\mathrm{H}$

$22 \mathrm{R}^{1}=$ sec-Butyl, $\mathrm{R}^{2}=\mathrm{OH}, \mathrm{R}^{3}=\mathrm{CH}_{3}$

$23 \mathrm{R}^{1}=$ sec-Butyl, $\mathrm{R}^{2}=\mathrm{H}, \mathrm{R}^{3}=\mathrm{CH}_{3}$

$24 R^{1}=$ sec-Butyl, $R^{2}=H, R^{3}=H$

$25 \mathrm{R}^{1}=\mathrm{CH}_{3}, \mathrm{R}^{2}=\mathrm{OH}, \mathrm{R}^{3}=\mathrm{CH}_{3}$

$26 \mathrm{R}^{1}=\mathrm{CH}_{3}, \mathrm{R}^{2}=\mathrm{H}, \mathrm{R}^{3}=\mathrm{CH}_{3}$

A

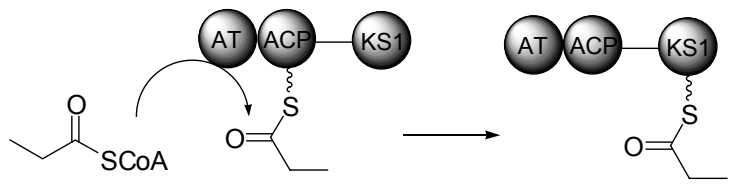

B

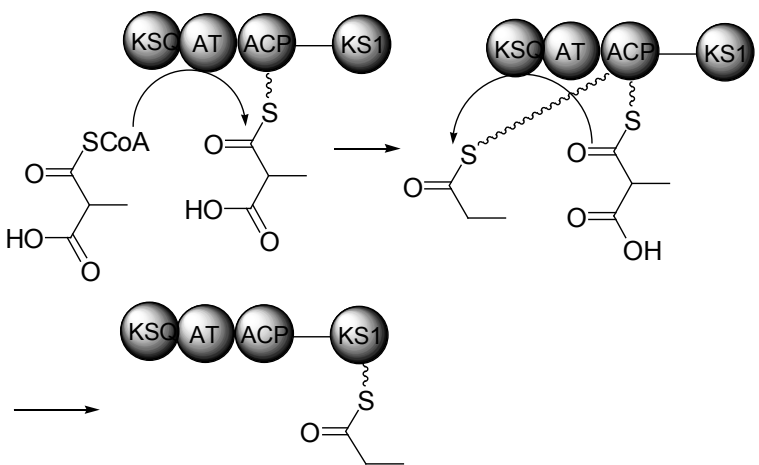

图 3 聚酮链起始单元的不同催化机制

Figure 3 Alternative mechanisms for polyketide chain initiation (A) Erythromycin DEBS chian initiation: transfer of propionate group from $\mathrm{CoA}$ to ACP. (B) Chain initiation on KSQ-containing loading modules: transfer methylmalonyl gourp from CoA to ACP then decarboxylation by $\mathrm{KSQ}^{[33]}$

把 oleandomycin 起始模块 KSQ 结构域与雷帕需素 rapamycin 延伸模块 2 中的 AT2 结构域(识别丙二酰辅酶 A)相连, 用此结构域替换红霉素 PKS 起始模块的 AT 结 构域(识别丙酰辅酶 A), 能够使该杂合模块最终把丙酰 基传递到红霉素 PKS 第一个延伸模块的酮基硫酯合成 酶 $\mathrm{KS}$ 上, 从而产生内酯环 C-13 带有甲基的红需素类似 物 $25, \mathbf{2 6}^{[33]}$.

\section{2 红雫素 PKS 结构修饰功能域的改变}

在 I 型 PKS 的某些模块组合有 $\beta$-酮基还原酶 $\mathrm{KR}$ 、 脱水酶 $\mathrm{DH}$ 和烯醇还原酶 ER 等功能域, 这些功能域非 PKS 催化反应往下延伸的必须基团，但它们的存在会影 响大环骨架上 $\beta$-羰基的还原水平. 这些结构域的功能如 Scheme 1 所示 ${ }^{[34]}$.
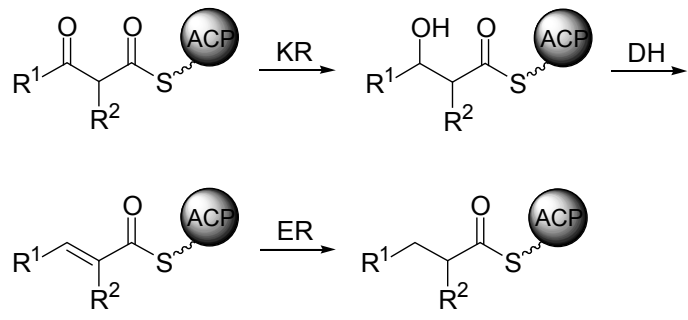

Scheme 1

分别对红霉素 PKS 延伸模块 6 中酮基还原酶 KR 功 能域和模块 4 中烯醇还原酶 ER 功能域失活, 可以得到 相应的 C-3 酮基类似物 27 和 C-6-C-7 不饱和类似物 $\mathbf{2 8}^{[35,36]}$. 此外, 直接删除红霉素 PKS 延伸模块 6 中酮基 还原酶 KR 结构域将影响与之相连的 AT 结构域的底物 识别特异性，结果除了产生化合物 27 外，还产生预料不 到的化合物 29 $^{[23]}$. 对红霉素 PKS 唯一 DH(延伸模块 4) 活性中心的点突变, 将导致 DEBS 蛋白的完全失活 ${ }^{[37]}$, 可见组合生物合成过程中基因操纵的方法也起关键作 用.

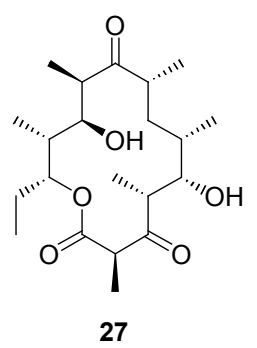

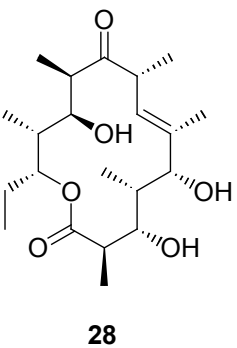

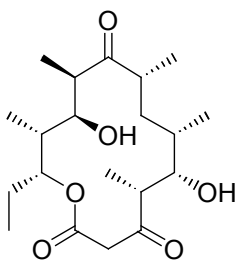

29

除了对这些功能域的删除, 在红霉素的 PKS 中增 加相关的功能域，同样能产生红霉素的一些结构类似 物. McDaniel 等采用合成雷帕霉素 PKS 延伸模块 4 的 $\mathrm{DH} / \mathrm{KR}$ 结构域分别替换红霉素 PKS 延伸模块 2、模块 6 中的 $\mathrm{KR}$ 结构域, 分别产生内酯环上含有烯键的化合物 30，31. 而采用雷帕霉素 PKS 中的延伸模块 1 的 $\mathrm{DH} / \mathrm{ER} / \mathrm{KR}$ 结构域分别替换红霉素 PKS 延伸模块 2、模 块 5 中的 $\mathrm{KR}$ 结构域则产生内酯环脱羟基的化合物 32, 33. 但令人意外的是采用此结构域替换红霉素 PKS 延 伸模块 6 时，并未发现脱羟基化合物 34 出现，反而有较

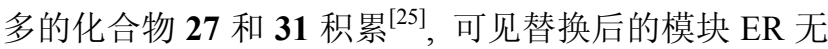
活性, KR 功能不完全. 这点可以反映插入模块的功能受 整体结构的影响. 同时基于这个酶学催化基础，作者把 
各种聚酮类的结构域或模块互相组合, 分别得到多种组 合突变的重组子, 表达得到近 60 种 6-脱氧红霉内酯类 似物库. 这占当时已知的所有聚酮化合物数量的 $3 \%$, 而且已经超过之前自然界所发现的不同大环内酯环结 构的总量. 值得注意的是, 这些复杂的结构类似物采用 化学合成的方法都是很难获得的. 这些研究充分体现了 组合生物合成在扩展天然产物结构多样性方面的巨大 潜力.
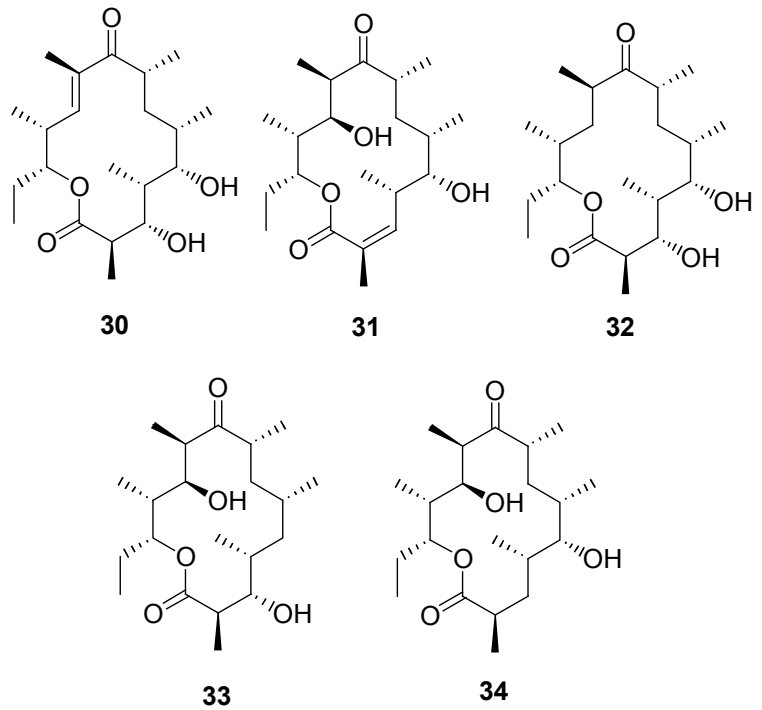

\section{3 内酯环单元数的改变}

红霉素大环内酯合成单元链完成延伸后在 DEBS3 的羟基端有一个硫酯酶酶域 TE, 负责将合成的长链脂 肪酸从 PKS 上水解下来, 并与 PKS 的其它部位共同作 用, 将产物环化成一个十四元环的化合物 6-脱氧红霉内 酯 ${ }^{[38]}$. 硫酷酶酶域 TE 对聚酮链的长度有较高的容忍性, 能够识别环化不同数目碳链原子形成数目不同元环. 如 把 TE 结构域连到 DEBS1 的羟基末端能够产生 6 元环内 酯化合物 $\mathbf{3 5}^{[39]}$. 有趣的是直接把含有红霉素合成延伸 模块 5 和模块 6 及 $\mathrm{TE}$ 的 DEBS3 基因导入到天蓝色链霉 菌 S. coelicolor CH999 中, 在不含 loading domain 的情况 下, 该蛋白识别甲基丙二酸单酰辅酶 $\mathrm{A}$ 为底物脱羧形成 丙酰基往下继续延伸、环化形成 6 元环内酯 $\mathbf{3 6}^{[40]}$. 这种 合成机制与前面所述起始模块含有 KRQ 结构域的催化 机制相似(图 3). 化合物 $\mathbf{3 6}$ 的构型与 $\mathbf{3 5}$ 不同在于 $\beta$-酮基 还原酶 KR 功能域催化的立体选择性: 红霉素合成延伸 模块 1 的 $\mathrm{KR}_{1}$ 功能域把聚酮中间产物 $\beta$-酮基催化还原成 羟基时专一地产生 $R$ 构型产物, 而其它模块的 $\beta$-酮基还 原酶如 $\mathrm{KR}_{2}, \mathrm{KR}_{5}, \mathrm{KR}_{6}$ 都产生 $S$ 构型的产物 ${ }^{[1,42]}$. $\mathrm{KR}$ 功 能域属于一类短链还原酶(short-chain dehydrogenase/reductase, SDR)家族的酶系 ${ }^{[43]}$, 此家族酶系活性位点中含 有非常保守的赖氨酸、丝氨酸及酪氨酸残基, 活性中心
的结构具有较高的相似性. 但催化产生 $R$ 构型化合物的 $\mathrm{KR}$ 活性中心一侧含有一个高度保守的天冬氨酸残基, 而还原产生 $S$ 构型化合物的 KR 活性中心则缺少此天冬 氨酸残基, 但在活性中心相反的一侧含有一个色氨酸残 基. 这些活性中心结构上细微差异使聚酮类化合物(底 物)进入 KR 活性中心时的方向不同, 从而最终使还原产 物形成不同的立体构型 ${ }^{[36,41,44]}$. 把 $\mathrm{TE}$ 结构域连到延伸 模块 5 的羟基端能够产生 12 元环内酯 ${ }^{[45]}$, 把合成雷帕 霉素 rapamycin PKS 模块 2 或模块 5 插入到红霉素 PKS 延伸模块 1 后面后, 能够产生 16 元环化合物 $\mathbf{3 8 ,}, \mathbf{3 9}$ 及在 C-13 位环化的十四元环产物 $\mathbf{4 0 ,} \mathbf{4 1}^{[46]}$.

\section{4 后修饰途径过程中的组合生物合成}

红霉素生物合成的后修饰过程包括在 6-脱氧红霉 内酯环上羟基化、糖基化以及在糖基上的甲基化. 对这 些修饰途径关键基因的敲除，都会导致前面中间代谢产 物的积累, 从而在细胞中合成红霉素 $\mathrm{A}$ 的一系列类似物 (化合物 6 11). 目前人们在红霉素合成后修饰途径组 合生物合成中最感兴趣的是内酯环的糖基化, 这些糖基 是红霉素生物活性的关键所在. 有报道显示德胺糖 (desosamine) 存在于所有 14 元大环内酯活性天然产物 中, 是红需素与核糖体接合从而抑制细菌生长的一个关 键因素 ${ }^{[23,47]}$. C-3 上的 $L$-碳霉糖(mycarose)并不是红霉素 抗菌活性所必须的，而且去除以后的红霉素衍生物对一 些原本对红霉素有耐药性的细菌产生抗性 ${ }^{[48]}$. 这些发 现都显示着糖基在红霉素生物活性上的重要作用, 因此 糖基的改变也成为红霉素组合生物合成研究的一个热 点. 糖基转移酶 EryBV 催化红霉素内酯环 C-3 羟基上的 糖基化, 对其体外测活实验表明, EryBV 能够识别一系 列其它的糖基作为底物合成红霉素类似物, 虽然目前从 催化效率上看还比较低 ${ }^{[49]}$.

糖基转移酶 EryCIII 催化 C-5 羟基上的糖基化, 相 对而言有较高的底物特异性 ${ }^{[50]}$. 但目前仍然有报道显 示 EryCIII 能够把 $D$-mycaminose 转移到内酯环的 C-5 差基上从而形成新的红霉素类似物红霉素 $\mathrm{M}(\mathbf{5 3})^{[51]}$, 此 外在缺乏底物 $D$-desosamine 时, EryCIII 还能再识别一个 mycarose 催化形成化合物 $\mathbf{5 4}^{[52]}$.

某些外源的糖基转移酶对底物容忍性比较高, 能够 将糖基转移至红霉素内酯环上. 如来自 S. eurythermus 的 AngMII 是泰乐菌素合成中糖基化酶, 利用 tylactone (55)作为天然底物在 C-5 羟基上糖基化 $D$-mycaminose 产生化合物 56 (Eq. 1). 研究发现 AngMII 糖基化酶能够 识别以十四元环的 erythronolide B (7)为底物, 催化合成 化合物 57 (Eq. 2) ${ }^{[52]}$. 令人意外的是该酶在十六元环中 糖基化的位置是 C-5 的羟基, 可是在红需素 14 元环中糖 基化的位置却是 $\mathrm{C}-3$ 的羟基. 
A

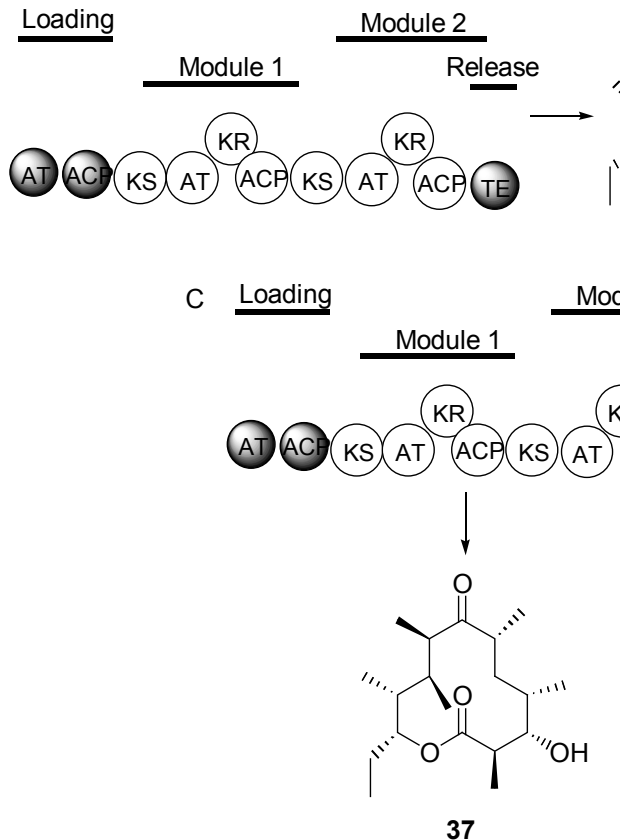

B

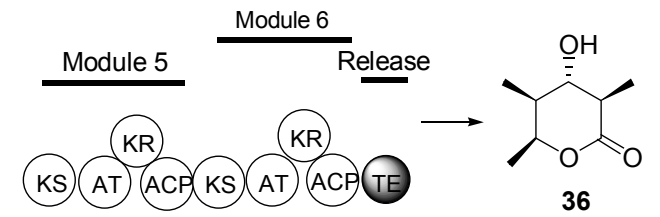

KS AT ACP KS AT ACP TE
35<smiles>CC1OC(=O)C(C)C(C)C1O</smiles>

RR)

Module 3

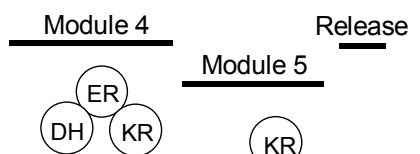

DH $\mathrm{KR}$

CP $\mathrm{KS}$ AT ACP KS AT ACP KS AT ACP TE<smiles></smiles>

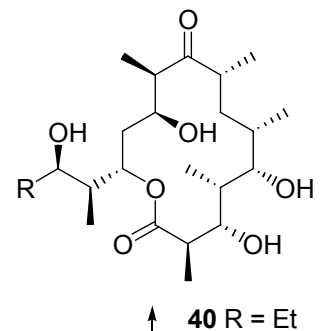

$39 \mathrm{R}=\mathrm{Me}$ $41 \mathrm{R}=\mathrm{Me}$

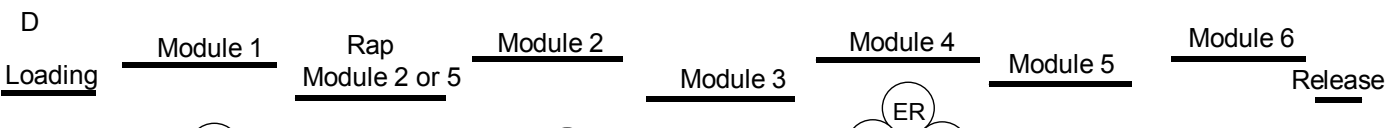

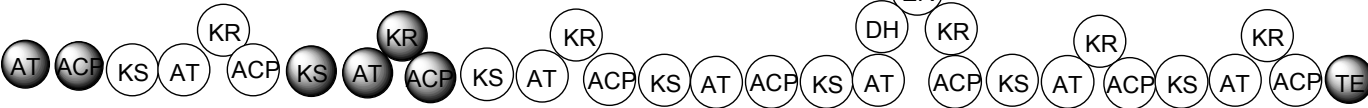

图 4 杂合 PKS 的结构与其催化合成的产物

Figure 4 Domain organization of hybrid PKSs and their products

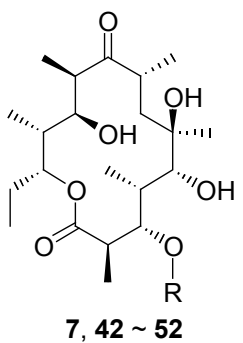

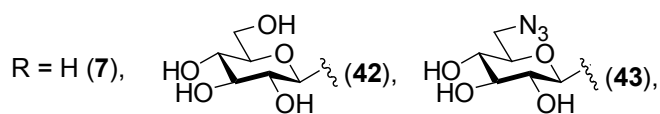

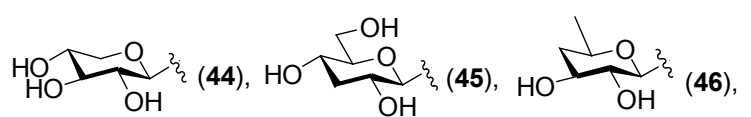

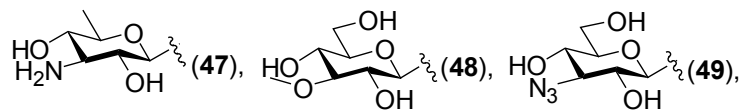

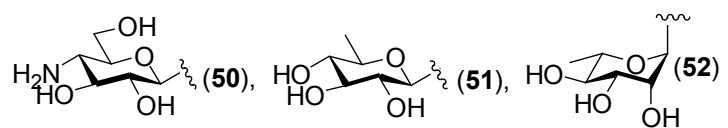

还有报道显示，与红需素结构非常相似的另外一个 天然产物苦霉素 picromycin C-5 羊基的德胺糖转移酶对 底物的容忍性也比较宽泛, 能够识别一系列 6-脱氧红霉

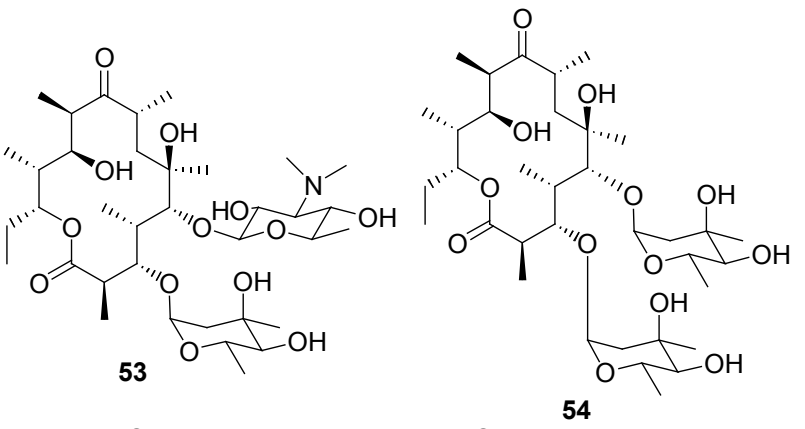

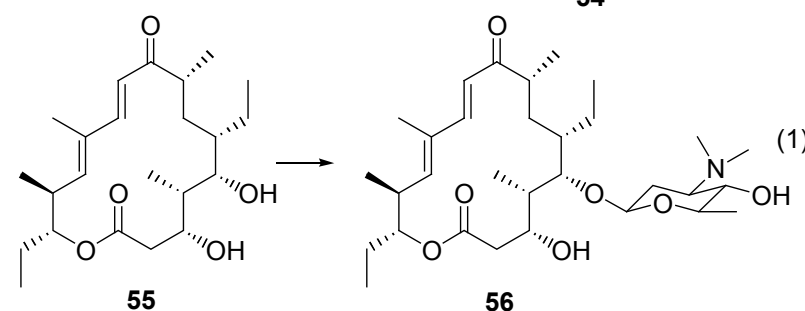

56 
内酯的结构类似物. 用这种方法可以合成超过 20 种以 上的新化合物 ${ }^{[53]}$.

\section{3 问题和展望}

在新药的研发领域, 通过组合生物合成优化先导化 合物的结构与随机合成化合物库篮选相比具有明显的 优势. 综合使用上述方法对红霉素进行组合生物合成理 论上几乎可以产生无数种红霉素的结构类似物. 但是在 实际操作过程中新化合物能否被催化合成取决于酶对 于底物的容忍性. 一个不容忽视的问题是绝大多数经过 组合生物合成手段合成的结构类似物产量要比原始的 天然产物低, 很多情况得到目标产物的量甚至没有利用 价值. 产生这种现象的根本原因在于酶的结构与底物的 关系发生改变. 在自然界中存在的酶催化反应都是由千 百万年进化而来, 天然的酶与天然的底物在进化过程中 已经处于一个相对最适的状态. 一方面通过人为的方法 产生新杂合酶从概率上来说是很难比天然的酶对底物 更加匹配. 另一方面由新的杂合酶催化合成的新化合物 也很难比天然的代谢中间产物更易被后续合成途径的 酶所识别. 解决这个问题可以从两个思路出发: 一个思 路是从酶结构与功能的关系考虑, 在构建杂合酶的过程 中尽量对酶的结构破坏最小. 采用关键活性位点突变的 方法对 I 型 PKS 中某个功能域的失活能减少对聚酮合酶 整体结构的破坏, 获得较高产量的目标产物 ${ }^{[34]}$. 在功能 域之间的替换或者插入过程中, 新的结构域与原来结构 域之间连接边界的选择是杂合酶有否有活性的关键所 在, 这主要是因为新插入或者删除的肽段对整体蛋白结 构的破坏 ${ }^{[23]}$. 这种破坏更多的体现在蛋白质三级结构 的破坏上，有报道显示利用合成苦霉素 PKS 的 pikAI, pikAII 亚基与合成红霉素 PKS 的 DEBS3 亚基形成组合 蛋白 ${ }^{[54]}$, 要比直接在红霉素 PKS 的 DEBS2 进行功能域 替换产生的杂合蛋白 ${ }^{[25]}$ 催化产生化合物 $\mathbf{5 8}$ 的量高出 100 多倍. 第二个思路是从酶催化反应动力学的角度出 发, 增加反应体系中酶和底物的浓度. 在生物合成的过 程中, 反应体系即细胞宿主. 改善细胞中红霉素合成前 体底物一一丙酰辅酶 $\mathrm{A}$ 及甲基丙二酸单酰辅酶 $\mathrm{A}$ 的供 应量 ${ }^{[55,56]}$, 能够显著提高红雼素的产量. 同时, 采用红 霉素高产菌株作为宿主来生产红霫素的结构类似物, 目 标产物的量同样有明显提高 ${ }^{[57]}$. 由于在大肠杆菌中可 以方便的进行基因操作, 强化外源蛋白的表达以及控制 前体合成, 也是一个较佳的组合生物合成宿主. 最近红 霉素 $\mathrm{A}$ 及其组合生物合成得到的结构类似物在大肠杆 菌内异源表达成功, 这为 I 型 PKS 聚酮类化合物的生物 合成开辟了一条新的道路 ${ }^{[58]}$.

目前第二代、第三代红霉素药物是人类使用最为广

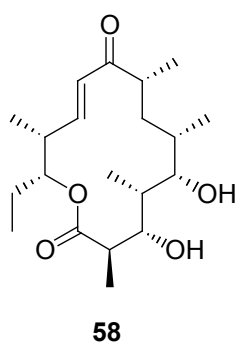

泛的抗生素之一, 需求量巨大. 这些药物的合成都是以 红霉素 A 为原料、通过对大环内酯骨架的化学修饰来获 得的, 工艺较为复杂, 容易造成环境污染. 但是从化学 结构来看, 红霉素 A (1) 并非是这些药物 2 5 化学合成 的最佳起始物. 由于红霉素 $\mathrm{A}$ 可以从细菌发酵液里直接 提取, 是目前市场上唯一可以提供的 14 元大环内酯类 大宗原料药, 所有红霉素类药物的化学合成均由红霉素 $\mathrm{A}$ 衍生而来. 采用组合生物合成的方法在细胞中生产更 佳的红霉素类药物中间体或者直接生产新一代的红霉 素将是开发红霉素新合成路线的新思路. 综上所述，随 着组合生物合成技术的成功应用, 化学合成与生物合成 将更加紧密地联系在一起, 从而促进新药的开发和发 展, 成为人类战胜各种疾病的有力武器.

\section{References}

[1] Demain, A. L. Appl. Microbiol. Biotechnol. 1999, 52, 455.

[2] Spizek, J.; Novotna, J.; Rezanka, T.; Demain, A. L. J. Ind. Microbiol. Biotechnol. 2010, 37, 1241.

[3] Walsh, C.; Wright, G. Chem. Rev. 2005, 105, 391.

[4] Li, J. W.; Vederas, J. C. Science 2009, 325, 161.

[5] Newman, D. J.; Cragg, G. M.; Snader, K. M. J. Nat. Prod. 2003, 66,1022 .

[6] Shen, B.; Liu, W.; Nonaka, K. Curr. Med. Chem. 2003, 10, 2317.

[7] Walsh, C. T. ChemBioChem 2002, 3, 125.

[8] McGuire, J. M.; Bunch, R. L.; Anderson, R. C.; Boaz, H. E.; Flynn, E. H.; Powell, H. M.; Smith, J. W. Schweiz Med. Wochenschr. 1952, 82, 1064.

[9] Bosnar, M.; Kelneric, Z.; Munic, V.; Erakovic, V.; Parnham, M. J. Antimicrob. Agents Chemother. 2005, 49, 2372.

[10] Ma, Z.; Nemoto, P. A. Curr. Med. Chem.-Anti-Infective Agents 2002, 1,15 .

[11] Staunton, J.; Wilkinson, B. Chem. Rev. 1997, 97, 2611.

[12] Savino, C.; Montemiglio, L. C.; Sciara, G.; Miele, A. E.; Kendrew, S. G.; Jemth, P.; Gianni, S.; Vallone, B. J. Biol. Chem. 2009, 284, 29170.

[13] Cupp-Vickery, J. R.; Poulos, T. L. Nat. Struct. Biol. 1995, 2, 144.

[14] Keatinge-Clay, A. J. Mol. Biol. 2008, 384, 941.

[15] Oliynyk, M.; Samborskyy, M.; Lester, J. B.; Mironenko, T.; Scott, N.; Dickens, S.; Haydock, S. F.; Leadlay, P. F. Nat. Biotechnol. 2007, 25, 447.

[16] Shen, B. Curr. Opin. Chem. Biol. 2003, 7, 285.

[17] Donadio, S.; Staver, M. J.; McAlpine, J. B.; Swanson, S. J.; Katz, L. Science 1991, 252, 675.

[18] Staunton, J.; Weissman, K. J. Nat. Prod. Rep. 2001, 18, 380.

[19] Weber, J. M.; Leung, J. O.; Swanson, S. J.; Idler, K. B.; McAlpine, J. B. Science 1991, 252, 114. 
[20] Lambalot, R. H.; Cane, D. E.; Aparicio, J. J.; Katz, L. Biochemistry 1995, 34,1858

[21] Chen, Y.; Deng, W.; Wu, J.; Qian, J.; Chu, J.; Zhuang, Y.; Zhang, S.; Liu, W. Appl. Environ. Microbiol. 2008, 74, 1820.

[22] Wu, J.; Zhang, Q.; Deng, W.; Qian, J.; Zhang, S.; Liu, W. Appl. Environ. Microbiol. 2011, 77, 7508.

[23] McDaniel, R.; Welch, M.; Hutchinson, C. R. Chem. Rev. 2005, 105, 543.

[24] Ruan, X.; Pereda, A.; Stassi, D. L.; Zeidner, D.; Summers, R. G.; Jackson, M.; Shivakumar, A.; Kakavas, S.; Staver, M. J.; Donadio, S.; Katz, L. J. Bacteriol. 1997, 179, 6416.

[25] McDaniel, R.; Thamchaipenet, A.; Gustafsson, C.; Fu, H.; Betlach, M.; Ashley, G. Proc. Natl. Acad. Sci. U. S. A. 1999, 96, 1846.

[26] Lu, L.; Arinthip, T.; Hong, F. J. Am. Chem. Soc. 1997, 119, 10553.

[27] Petkovic, H.; Lill, R. E.; Sheridan, R. M.; Wilkinson, B.; McCormick, E. L.; McArthur, H. A.; Staunton, J.; Leadlay, P. F.; Kendrew, S. G. J. Antibiot. (Tokyo) 2003, 56, 543.

[28] Stassi, D. L.; Kakavas, S. J.; Reynolds, K. A.; Gunawardana, G.; Swanson, S.; Zeidner, D.; Jackson, M.; Liu, H.; Buko, A.; Katz, L. Proc. Natl. Acad. Sci. U. S. A. 1998, 95, 7305.

[29] Marsden, A. F.; Wilkinson, B.; Cortes, J.; Dunster, N. J.; Staunton, J.; Leadlay, P. F. Science 1998, 279, 199.

[30] Cundliffe, E.; Bate, N.; Butler, A.; Fish, S.; Gandecha, A.; Merson-Davies, L. Antonie Van Leeuwenhoek 2001, 79, 229.

[31] Xue, Y.; Zhao, L.; Liu, H. W.; Sherman, D. H. Proc. Natl. Acad. Sci. U. S. A. 1998, 95, 12111.

[32] Waldron, C.; Matsushima, P.; Rosteck, P. R., Jr.; Broughton, M. C.; Turner, J.; Madduri, K.; Crawford, K. P.; Merlo, D. J.; Baltz, R. H. Chem. Biol. 2001, 8, 487.

[33] Long, P. F.; Wilkinson, C. J.; Bisang, C. P.; Cortes, J.; Dunster, N.; Oliynyk, M.; McCormick, E.; McArthur, H.; Mendez, C.; Salas, J. A.; Staunton, J.; Leadlay, P. F. Mol. Microbiol. 2002, 43, 1215.

[34] Katz, L.; McDaniel, R. Med. Res. Rev. 1999, 19, 543.

[35] Donadio, S.; McAlpine, J. B.; Sheldon, P. J.; Jackson, M.; Katz, L. Proc. Natl. Acad. Sci. U. S. A. 1993, 90, 7119.

[36] Reid, R.; Piagentini, M.; Rodriguez, E.; Ashley, G.; Viswanathan, N.; Carney, J.; Santi, D. V.; Hutchinson, C. R.; McDaniel, R. Biochemistry 2003, 42, 72.

[37] Bevitt, D. J.; Staunton, J.; Leadlay, P. F. Biochem. Soc. Trans. 1993, 21, 30S.

[38] Gokhale, R. S.; Hunziker, D.; Cane, D. E.; Khosla, C. Chem. Biol. 1999, 6, 117.

[39] Kao, C. M.; Luo, G.; Katz, L.; Cane, D. E.; Khosla, C. J. Am.
Chem. Soc. 1994, 116, 11612.

[40] Jacobsen, J. R.; Cane, D. E.; Khosla, C. Biochemistry 1998, 37, 4928.

[41] Cane, D. E. J. Biol. Chem. 2010, 285, 27517.

[42] Siskos, A. P.; Baerga-Ortiz, A.; Bali, S.; Stein, V.; Mamdani, H.; Spiteller, D.; Popovic, B.; Spencer, J. B.; Staunton, J.; Weissman, K. J.; Leadlay, P. F. Chem. Biol. 2005, 12, 1145.

[43] Kallberg, Y.; Oppermann, U.; Jornvall, H.; Persson, B. Eur. J. Biochem. 2002, 269, 4409.

[44] Keatinge-Clay, A. T.; Stroud, R. M. Structure 2006, 14, 737.

[45] Kao, C. M.; Luo, G.; Katz, L.; Cane, D. E.; Khosla, C. J. Am. Chem. Soc. 1995, 117, 9105.

[46] Rowe, C. J.; Bohm, I. U.; Thomas, I. P.; Wilkinson, B.; Rudd, B. A.; Foster, G.; Blackaby, A. P.; Sidebottom, P. J.; Roddis, Y.; Buss, A. D.; Staunton, J.; Leadlay, P. F. Chem. Biol. 2001, 8, 475.

[47] Schlunzen, F.; Zarivach, R.; Harms, J.; Bashan, A.; Tocilj, A.; Albrecht, R.; Yonath, A.; Franceschi, F. Nature 2001, 413, 814.

[48] Agouridas, C.; Denis, A.; Auger, J. M.; Benedetti, Y.; Bonnefoy, A.; Bretin, F.; Chantot, J. F.; Dussarat, A.; Fromentin, C.; D'Ambrieres, S. G.; Lachaud, S.; Laurin, P.; Le Martret, O.; Loyau, V.; Tessot, N. J. Med. Chem. 1998, 41, 4080.

[49] Zhang, C.; Fu, Q.; Albermann, C.; Li, L.; Thorson, J. S. ChemBioChem 2007, 8, 385.

[50] Lee, H. Y.; Chung, H. S.; Hang, C.; Khosla, C.; Walsh, C. T.; Kahne, D.; Walker, S. J. Am. Chem. Soc. 2004, 126, 9924.

[51] Yuan, Y.; Chung, H. S.; Leimkuhler, C.; Walsh, C. T.; Kahne, D.; Walker, S. J. Am. Chem. Soc. 2005, 127, 14128.

[52] Schell, U.; Haydock, S. F.; Kaja, A. L.; Carletti, I.; Lill, R. E.; Read, E.; Sheehan, L. S.; Low, L.; Fernandez, M. J.; Grolle, F.; McArthur, H. A.; Sheridan, R. M.; Leadlay, P. F.; Wilkinson, B.; Gaisser, S. Org. Biomol. Chem. 2008, 6, 3315.

[53] Tang, L.; McDaniel, R. Chem. Biol. 2001, 8, 547.

[54] Tang, L.; Fu, H.; McDaniel, R. Chem. Biol. 2000, 7, 77.

[55] Reeves, A. R.; Brikun, I. A.; Cernota, W. H.; Leach, B. I.; Gonzalez, M. C.; Mark Weber, J. Metab. Eng. 2007, 9, 293.

[56] Reeves, A. R.; Brikun, I. A.; Cernota, W. H.; Leach, B. I.; Gonzalez, M. C.; Weber, J. M. J. Ind. Microbiol. Biotechnol. 2006, 33,600 .

[57] Rodriguez, E.; Hu, Z.; Ou, S.; Volchegursky, Y.; Hutchinson, C. R.; McDaniel, R. J. Ind. Microbiol. Biotechnol. 2003, 30, 480.

[58] Zhang, H.; Wang, Y.; Wu, J.; Skalina, K.; Pfeifer, B. A. Chem. Biol. 2010, 17, 1232 . 